\title{
The Impact of Selfishness in Hypergraph Hedonic Games
}

\author{
Alessandro Aloisio, ${ }^{1,2}$ Michele Flammini, ${ }^{2}$ Cosimo Vinci $^{2}$ \\ ${ }^{1}$ University of L'Aquila, L'Aquila, Italy \\ ${ }^{2}$ Gran Sasso Science Institute, L'Aquila, Italy \\ alessandro.aloisio@univaq.it, michele.flammini@gssi.it, cosimo.vinci@gssi.it
}

\begin{abstract}
We consider a class of coalition formation games that can be succinctly represented by means of hypergraphs and properly generalizes symmetric additively separable hedonic games. More precisely, an instance of hypegraph hedonic game consists of a weighted hypergraph, in which each agent is associated to a distinct node and her utility for being in a given coalition is equal to the sum of the weights of all the hyperedges included in the coalition. We study the performance of stable outcomes in such games, investigating the degradation of their social welfare under two different metrics, the $k$-Nash price of anarchy and $k$-core price of anarchy, where $k$ is the maximum size of a deviating coalition. Such prices are defined as the worst-case ratio between the optimal social welfare and the social welfare obtained when the agents reach an outcome satisfying the respective stability criteria. We provide asymptotically tight upper and lower bounds on the values of these metrics for several classes of hypergraph hedonic games, parametrized according to the integer $k$, the hypergraph arity $r$ and the number of agents $n$. Furthermore, we show that the problem of computing the exact value of such prices for a given instance is computationally hard, even in case of non-negative hyperedge weights.
\end{abstract}

\section{Introduction}

In several real-life scenarios arising from economics, politics, and sociology, we assist to phenomena of coalition formation, in which each person, denoted as agent, forms coalitions with other agents to get some benefit, experiencing a utility that depends on the particular set of agents she joins to. A simple and efficient model that formally describes such phenomena is that of hedonic games (Drèze and Greenberg 1980; Banerjee, Konishi, and Sönmez 2001; Bogomolnaia and Jackson 2002), which constitute an interesting class of general coalition formation games, in which agents mind only their coalitions, regardless of how the others aggregate. In hedonic games, we have a finite set of agents, each in turn having a preference order on all the possible coalitions containing her.

A very interesting class of hedonic games is that of symmetric additively separable hedonic games (SASHGs), in

Copyright (C) 2020, Association for the Advancement of Artificial Intelligence (www.aaai.org). All rights reserved. which each couple of agents is associated to a weight determining their level of appreciation for being together, and the overall utility of each agent is simply the sum of the individual values she assigns to the members of her own coalition. Such games can be suitably described by undirected weighted graphs, in which nodes represent agents, and weighted edges represent couples of agents with their appreciation. Besides their nice property of being succinctly representable, SASHGs attracted research attention because they are able to capture several realistic coalition formation scenarios. However, they cannot handle other real life situations in which coalitions are worth if they reach some critical mass, as in the formation of political parties. Indeed, several politicians may be mutually incompatible, but forming coalitions with more than two people can increase the success of each member; thus, we need subsets of more than two people with positive weight together with edges having negative weights (which model the level of incompatibility of each couple of politicians). Another example comes from team formation, where one has to form groups of workers that have the set of abilities required to perform some tasks, without which the group is unsuccessful. In this case we need to associate a positive weight to each group of workers able to perform a task when working together, and a weight to each edge modelling the level of compatibility of each couple of workers.

In light of the above examples (and many others), the graph structure of SASHGs may be not sufficient to describe several real-life phenomena of coalition formation. To cope with this issue, we consider a superclass of SASHGs that, while maintaining a succinct representation, can capture the above more general scenarios. Namely, we focus on the class of $r$-hypergraph hedonic games ( $r$-HHGs or simply HHGs), in which each subset of at most $r \geq 1$ agents is associated to a weight determining the level of liking for being a group included in the same coalition, and the utility of each agent is the sum of the values she assigns to the groups in her coalition. Such games can be completely described by weighted hypergraphs, in which again nodes represent agents, while weighted hyperedges represent groups of agents with their valuation. As already observed, $r$-HHGs are a generalization of SASHGs, and a proper subclass of synchronization 
games on hypergraphs (Simon and Wojtczak 2017).

Depending on the characteristics of the agents (e.g., their willingness to cooperate with each other), the selfish behaviour can lead to stable coalitions, in which no agent can improve her utility according to some deviation criteria. The stability concepts we consider are the $k$-Nash stability and the $k$-core stability. In a $k$-Nash stable outcome, no subset of at most $k$ agents can move into other existing or new coalitions in such a way that each agent of the considered subset improves her utility. Similarly, in a $k$-core stable outcome, no subset of at most $k$ agents can create a new coalition, in such a way that each deviating agent improves. These stability criteria are strictly connected with the known concepts of core stability and Nash equilibrium (Osborne 2004; Chalkiadakis, Elkind, and Wooldridge 2011), widely considered in artificial intelligence in the context of cooperative and non-cooperative games, and also investigated in hedonic games (Aziz, Brandt, and Seedig 2013; Gairing and Savani 2010).

Besides stability, one desirable property in coalition formation is guaranteeing a general level of appreciation or social welfare, reasonably defined as the sum of all the agents' utilities. However, if there is no central authority (such as a Governmental Entity) deciding how to form the coalitions, the selfish behaviour of the agents may lead to stable outcomes that are suboptimal, i.e., with a low social welfare. To measure this inefficiency, several metrics have been proposed. A widely used one in game theory is the price of anarchy (Koutsoupias and Papadimitriou 1999), defined as the highest ratio between the social welfare of an optimal outcome, and the one of a Nash stable outcome. This and similar metrics have been recently considered to measure the inefficiency also of some classes of hedonic games under different stability criteria (Elkind, Fanelli, and Flammini 2016; Kaklamanis, Kanellopoulos, and Papaioannou 2016; Bilò et al. 2018; Monaco, Moscardelli, and Velaj 2019).

\section{Our contribution}

In this work, we analyse the performance of stable outcomes in $r$-HHGs by resorting to the following metrics: (i) the $k$ Nash price of anarchy $\left(\mathrm{NPoA}_{k}\right)$, defined as the highest ratio between the social welfare of an optimal outcome and the one of a $k$-Nash stable outcome, and (ii) the $k$-core price of anarchy $\left(\mathrm{CPoA}_{k}\right)$, analogously defined with respect to $k$ core stable outcomes. We give an almost complete picture of the performance of such games in all the possible cases. In particular, we have obtained the following results:

- We provide almost tight bounds on the $\mathrm{CPoA}_{k}$ of standard $r$-HHGs with $k \geq r$ (Theorem 1, 2, and 3), where "standard" means that the hypergraph's weights satisfy some mild assumptions, allowing even the case of negative weights (unlike several works on the inefficiency of stable outcomes). Such bounds are parametric in $k, r$, the number of agents $n$, and are asymptotically tight in $\rho:=n / k$ if $r$ is constant.

- For all the cases of which we have not provided a finite upper bound, we show that the $\mathrm{CPoA}_{k}$ may be unbounded (Theorem 4 and 5).
- Some of the previous bounds hold even for the $\mathrm{NPoA}_{k}$ (Theorem 3 and Corollary 2). When dealing with nonnegative weights, we get a slightly better upper bound, holding also for $k=r-1$ (Theorem 6).

- For most of the cases for which we have not provided an upper bound, we show that the $\mathrm{NPoA}_{k}$ may be unbounded (Theorem 7 and 8).

See Table 1 and 2 for further details.

\begin{tabular}{|c||c|c|}
\hline & Standard & Non-Standard \\
\hline \hline$k \geq r$ & $\Theta\left(\rho^{r-1}\right)$ & $\infty$ \\
\hline$k<r$ & $\infty$ & $\infty$ \\
\hline
\end{tabular}

Table 1: $\mathrm{CPoA}_{k}$ of $r$-HHGs with $n$ agents w.r.t. $\rho:=n / k$.

\begin{tabular}{|c||c|c|c|}
\hline & Weights $\geq 0$ & Stand. & Non-Stand. \\
\hline \hline$k \geq r$ & $\Theta\left(\rho^{r-1}\right)$ & $\Theta\left(\rho^{r-1}\right)$ & $\infty$ \\
\hline$k=r-1$ & $\Theta\left(\rho^{r-1}\right)$ & $?$ & $\infty$ \\
\hline$k<r-1$ & $\infty$ & $\infty$ & $\infty$ \\
\hline
\end{tabular}

Table 2: $\mathrm{NPoA}_{k}$ of $r$-HHGs with $n$ agents w.r.t. $\rho:=n / k$.

Finally, regarding the time complexity of measuring the inefficiency of HHGs, we have shown that the problems of computing the $k$-core price of anarchy and the $k$-Nash price of anarchy of an $r$-HHG are NP-hard (Theorem 9 and 10).

To the best of our knowledge, this is the first work in which the inefficiency of coalition formation games succinctly representable by hypergraphs has been studied.

Due to lack of space, some proofs are sketched or omitted.

\section{Related work}

HHGs are related to many other well-known classes of games. In particular, they are a proper subclass of synchronization games, whose properties concerning the existence and computability of pure and strong Nash equilibria are studied in (Simon and Wojtczak 2017), without considering their inefficiency in terms of social welfare. Most of their results can be applied to show analogue ones in HHGs. Synchronization games extend polymatrix coordination games (Yanovskaya 1968; Cai and Daskalakis 2011), in which the agents utilities are pairwise separable, to hypergraphs. (Yanovskaya 1968; Cai and Daskalakis 2011; Rahn and Schäfer 2015; Apt et al. 2017) investigate the existence and computation of $k$-strong equilibria in these games and related variants, while (Rahn and Schäfer 2015; Apt et al. 2017; Feldman and Friedler 2015) consider the problem of measuring their inefficiency. Some of these inefficiency results can be applied to SASHGs with non negative weights, and are generalized in our work to the setting of hypergraphs with possibly negative weights.

Additively separable hedonic games (ASHGs) can be seen as a proper subclass of polymatrix coordination games, and their symmetric version (i.e., SASHGs), as already observed, is a proper sub-class of the games of HHGs. Due 
to their graph succinct representation, they are also connected with the graph games introduced by (Deng and Papadimitriou 1994). Properties guaranteeing the existence of core allocations (a core is a coalition structure in which no group of agents has an incentive to form a different coalition) are studied by (Banerjee, Konishi, and Sönmez 2001), while (Bogomolnaia and Jackson 2002), besides the core, consider other forms of stability, such as Nash and individual stability. (Olsen 2009) shows that deciding whether a Nash stable outcome of a ASHG exists is NP-complete, as well as deciding the existence of a non-trivial (different from the grand coalition) Nash stable outcome in SASHGs with non-negative weights. (Gairing and Savani 2010) prove that computing a Nash stable outcome in SASHGs is PLScomplete, while (Sung and Dimitrov 2010) show that in ASHGs determining the existence of a core stable, strict core stable, Nash stable, or individually stable outcome is NP-hard. (Aziz, Brandt, and Seedig 2013) show that computing a core or a strict core stable outcome is NP-hard even for symmetric and strict preferences. Furthermore, they show that the problem of computing optimal outcomes is NP-hard. As SASHGs are a subclass of HHGs, the results obtained in (Aziz, Brandt, and Seedig 2013) imply that the problem of computing a core stable outcome or an optimal outcome in HHGs is NP-hard, too. Furthermore, as strongly Nash stable outcomes are a subset of core stable outcomes, we also get that strongly Nash stable outcomes may not exist in HHGs. The problem of computing an outcome verifying particular optimality or stability criteria in ASHGs has been considered in (Aziz, Brandt, and Seedig 2011; Peters and Elkind 2015).

Another relevant class of hedonic games is that of fractional hedonic games, studied in (Aziz et al. 2019; Peters and Elkind 2015; Olsen 2012; Monaco, Moscardelli, and Velaj 2018; Carosi, Monaco, and Moscardelli 2019; Bilò et al. 2018; Flammini et al. 2018; Monaco, Moscardelli, and Velaj 2019). The inefficiency of these and other related classes of hedonic games under different stability and optimality notions has been considered in (Elkind, Fanelli, and Flammini 2016; Balliu et al. 2019; Balliu, Flammini, and Olivetti 2017; Flammini, Monaco, and Zhang 2017).

Finally, we remark that other games related to HHGs are the group activity selection games, studied in (Darmann et al. 2018; Igarashi, Bredereck, and Elkind 2017; Igarashi, Peters, and Elkind 2017; Bilò et al. 2019)), and max-cut games, studied in (Gourvès and Monnot 2009; Gourvès and Monnot 2010; Feldman and Friedler 2015; Carosi et al. 2019).

\section{Preliminaries}

Given two integers $r \geq 1$ and $n \geq 1$, let $[n]=\{1,2, \ldots, n\}$ and $(n)_{r}:=n \cdot(n-1) \cdot \ldots \cdot(n-r+1)$ be the falling factorial. Moreover, given a set $A$, let $\chi_{A}$ denote the indicator function, i.e., $\chi_{A}(x)=1$ if $x \in A, \chi_{A}(x)=0$ otherwise.

$r$-hypergraph hedonic games. A weighted hypergraph is a triple $\mathcal{H}=(V, E, w)$ consisting of a finite set $V=[n]$ of nodes, a collection $E \subseteq 2^{V}$ of hyperedges, and a weight $w: E \rightarrow \mathbb{R}$ associating a real value $w(e)$ with each hyperedge $e \in E$. For simplicity, when referring to weighted hypergraphs, we omit the term weighted.

The arity of a hyperedge $e$ is its size $|e|$. An $r$-hypergraph is a hypergraph such that the arity of each hyperedge is at most $r$, where $2 \leq r \leq n$. A complete $r$-hypergraph is a hypergraph $(V, E, w)$ such that $E:=\{U \subseteq V:|U| \leq r\}$. A uniform r-hypergraph (resp. weakly uniform $r$-hypergraph) is a hypergraph such that the arity of each hyperedge is $r$ (resp. either $r$, or 1). An undirected graph is a uniform 2hypergraph.

An $r$-hypergraph hedonic game $\mathcal{G}(\mathcal{H})(r$-HHG, or HHG when $r$ is not specified), is a strategic game based on an $r$ hypergraph $\mathcal{H}$, defined as follows:

Agents: The set of agents is $V=[n]$, i.e., each node corresponds to an agent. We reasonably assume that $n \geq 2$.

Strategy profiles: An outcome or coalition structure is a partition $C=\left\{C_{1}, C_{2}, \ldots, C_{h}\right\}$ of $V$. Each set $C_{l}$ is a coalition of $C$, and $h$ is the size of $C$.

Utility functions: Given a coalition structure $\boldsymbol{C}$ and an agent $i \in V$, let $C(i)$ be the coalition of $C$ such that $i \in C(i)$. Given $U \subseteq V$, let $E(U):=\{e \in E: e \subseteq U\}$. Given an agent $i \in V$, the utility $u_{i}(\boldsymbol{C})$ of $i$ in $\boldsymbol{C}$ is the sum of the weights of all the hyperedges $e$ containing $i$ such that $e \subseteq C(i)$, i.e., $u_{i}(\boldsymbol{C}):=\sum_{e \in E(C(i)): i \in e} w(e)$.

The grand coalition structure is the coalition structure defined as $C=\{V\}$, that is obtained when all agents belong to the same coalition. The singleton coalition structure is the coalition structure $C=\{\{1\},\{2\}, \ldots,\{n\}\}$, that is obtained when each coalition contains exactly one agent.

A complete r-hypergraph (resp. uniform r-hypergraph, resp. weakly uniform r-hypergraph, resp. graph) hedonic game is an $r$-HHG such that the underlying $r$-hypergraph is complete (resp. uniform, resp. weakly uniform, resp. a uniform 2-hypergraph). A standard $r$-hypergraph hedonic game is an $r$-HHG such that, for any $U \subseteq V$ with $|U| \geq 2$, we have that either (i) $\sum_{e \in E(U):|e|=s} \bar{w}(e) \geq 0$ for any $s \in[r] \backslash\{1\}$, or (ii) $\sum_{e \in E(U):|e|=s} w(e) \leq 0$ for any $s \in[r] \backslash\{1\}$.

Remark 1. The class of standard HHGs is well-motivated by real-life scenarios. Indeed, if we consider that a positive (resp. negative) weight of a hyperedge $e$ models the fact that all the agents belonging to $e$ are happy (resp. unhappy) to stay together in the same coalition, a HHG is standard if, given an arbitrary subset $U$ of agents, either they are happy on the average with respect to all the hyperedges of $U$ with arity $s>1$ (independently from $s$ ), or they are unhappy on the average with respect to such hyperedges.

Observe that weakly uniform $r$-HHGs and $r$-HHGs with non-negative weights are standard. In Figure 1, we exhibit a non-uniform game with both positive and negative weights that is standard. The social welfare $\operatorname{SW}(\boldsymbol{C})$ of a coalition structure $C$ is defined as the sum of all the agents' utilities in $\boldsymbol{C}$, i.e., $\operatorname{SW}(\boldsymbol{C}):=\sum_{i \in V} u_{i}(\boldsymbol{C})=$ $\sum_{l=1}^{h} \sum_{t=1}^{r} t \sum_{e \subseteq C_{l}:|e|=t} w(e)$. 


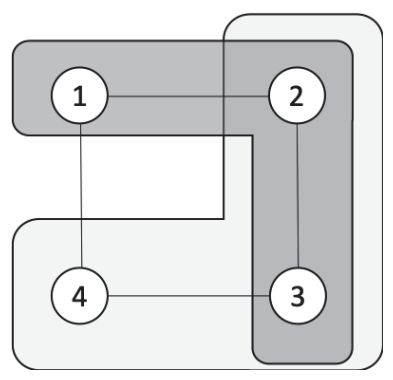

Figure 1: A hypergraph $\mathcal{H}$ with $V=[4]$ and $E=\{\{1,2\},\{2,3\},\{3,4\},\{4,1\},\{1,2,3\},\{2,3,4\}\}$, $w(\{1,2,3\})=w(\{2,3,4\})=3, w(\{1,2\})=$ $w(\{1,4\})=-1$, and $w(\{2,3\})=w(\{3,4\})=2$. $\mathcal{G}(\mathcal{H})$ is a standard 3-HHG. Indeed, if $U \in$ $\{\{1,2,3,4\},\{1,2,3\},\{2,3,4\},\{3,4,1\},\{2,3\},\{3,4\}\}$ we have that $\sum_{e \in E(U):|e|=s} w(e) \geq 0$ for any $s \in[r] \backslash\{1\}$, and if $U \in\{\{1,2,4\},\{1,2\},\{1,4\}\}$ we have that $\sum_{e \in E(U):|e|=s} w(e) \leq 0$ for any $s \in[r] \backslash\{1\}$.

$k$-Nash stability. Given a coalition structure $C=$ $\left\{C_{1}, C_{2}, \ldots, C_{h}\right\}$ and a subset of nodes $Z \subseteq V$, a function $\varphi: Z \rightarrow C \cup[n]$ is called deviation function of $Z$ from $\boldsymbol{C}$, and associates each agent of $Z$ with a coalition of $C$, or with an integer of $[n]$ that will be used to define a new coalition. Given $l \in[h]$ and $t \in[n]$, let $Z_{l}^{\prime}:=\varphi^{-1}\left[C_{l}\right]$ (i.e., the set of agents associated with coalition $C_{l}$ by function $\varphi$ ), and let $\hat{Z}_{t}:=\varphi^{-1}[t]$ (i.e., the set of agents associated with some new coalition by function $\varphi$ ). Given a deviation function $\varphi$ of $Z$ from $\boldsymbol{C}$, let $(\boldsymbol{C} \mid \varphi):=\left\{\left\{C_{1} \backslash Z\right\} \cup Z_{1}^{\prime},\left\{C_{2} \backslash Z\right\} \cup\right.$ $\left.Z_{2}^{\prime}, \ldots,\left\{C_{h} \backslash Z\right\} \cup Z_{h}^{\prime}, \hat{Z}_{1}, \hat{Z}_{2}, \ldots, \hat{Z}_{n}\right\} \backslash\{\emptyset\}$. Observe that $(\boldsymbol{C} \mid \varphi)$ defines a new coalition structure. A generic coalition structure $(\boldsymbol{C} \mid \varphi)$ is called deviation coalition structure of $Z$ from $C$, and it is equivalently defined as a coalition structure in which each agent $i$ of $Z$ moves from coalition $C(i)$ to coalition $\varphi(i)$ if $\varphi(i) \in \boldsymbol{C}$, and moves into a new coalition $\hat{Z}_{\varphi(i)}$ if $\varphi(i) \in[n]$, where $\hat{Z}_{\varphi(i)}$ is the coalition made of all the agents associated by $\varphi$ to the same integer $\varphi(i)$.

Given an integer $k \leq n$, a coalition structure $C$ is $k$-Nash stable if and only if, for any $Z \subseteq V$ with $|Z| \leq k$, and any deviation function $\varphi$ of $Z$ from $C$, there exists at least one agent $i \in Z$ such that $u_{i}(\boldsymbol{C}) \geq u_{i}(\boldsymbol{C} \mid \varphi)$. According to the definitions often adopted in several existing works on hedonic games, a 1-Nash stable coalition structure is equivalently called Nash stable, while an $n$-Nash stable coalition structure is called strongly Nash stable. For a game $\mathcal{G}(\mathcal{H})$, let $\mathrm{NS}_{k}(\mathcal{G}(\mathcal{H}))$ be the set of $k$-Nash stable coalition structures of $\mathcal{G}(\mathcal{H})$.

$k$-core stability. A deviation coalition structure $(\boldsymbol{C} \mid \varphi)$ of $Z$ from $C$ is called core deviation coalition structure of $Z$ from $C$ if $\varphi(i)=\emptyset$ for any $i \in Z$, and we equivalently denote it as $(\boldsymbol{C} \mid Z)$. Informally, a core deviation coalition structure is obtained when all the agents of set $Z$ move into the empty coalition, i.e., they form a new coalition made of all the agents of $Z$, only.
Given $k \leq n$, a coalition structure $C$ is $k$-core stable if and only if, for any $Z \subseteq V$ with $|Z| \leq k$, there exists at least one agent $i \in Z$ such that $u_{i}(\boldsymbol{C}) \geq \bar{u}_{i}(\boldsymbol{C} \mid Z)$. According to the definitions often adopted in several existing works on hedonic games, an $n$-core stable coalition structure is equivalently called core stable.

For a game $\mathcal{G}(\mathcal{H})$, let $\operatorname{CS}_{k}(\mathcal{G}(\mathcal{H}))$ be the set of $k$ core stable coalition structures of $\mathcal{G}(\mathcal{H})$. Observe that $\mathrm{NS}_{k}(\mathcal{G}(\mathcal{H})) \subseteq \mathrm{CS}_{k}(\mathcal{G}(\mathcal{H}))$

Remark 2. If $w(e) \geq 0$ for any $e \in E$ such that $|e|=1$, then each agent gets a non-negative utility in any $k$-core stable coalition structure (and thus, in any $k$-Nash stable coalition structure), otherwise she would deviate in a singleton coalition. Furthermore, the social optimum is guaranteed to be non-negative, as it achieves at least the social welfare of the singleton coalition structure.

In light of the previous remark and as commonly assumed in hedonic games, it is reasonable to suppose that for any agent is better staying alone than into a coalition in which she is unhappy; thus we do the following assumption:

Assumption 1. $w(e) \geq 0$ for any $e \in E$ such that $|e|=1$.

Quality of stable coalitions. For a game $\mathcal{G}(\mathcal{H})$, an optimal coalition structure $C^{*}$, also called social optimum, is an outcome maximizing the social welfare. The $k$-Nash price of anarchy of $\mathcal{G}(\mathcal{H})$ (denoted $\operatorname{NPoA}_{k}(\mathcal{G}(\mathcal{H}))$ ) is the worst-case ratio between the social welfare of a social optimum $C^{*}$, and the social welfare of a $k$-Nash stable coalition structure, that is, $\operatorname{NPoA}_{k}(\mathcal{G}(\mathcal{H})):=\max _{\boldsymbol{C} \in \mathrm{NS}_{k}(\mathcal{G}(\mathcal{H}))} \frac{\mathrm{SW}\left(\boldsymbol{C}^{*}\right)}{\mathrm{SW}(\boldsymbol{C})} \geq 1$, with the convention that $\infty:=c / 0$ for any $c>0,1:=0 / 0$, and $\mathrm{NPoA}_{k}(\mathcal{G}(\mathcal{H})):=1$ if $\mathcal{G}(\mathcal{H})$ does not admit $k$-Nash stable coalition structures. ${ }^{1}$ Analogously, the $k$-core price of anarchy of $\mathcal{G}(\mathcal{H})$ (denoted as $\mathrm{CPoA}_{k}(\mathcal{G}(\mathcal{H}))$ ) is the worst-case ratio between the social welfare of a social optimum $C^{*}$, and the social welfare of a $k$-core stable coalition structure, that is, $\mathrm{CPoA}_{k}(\mathcal{G}(\mathcal{H})):=\max _{\boldsymbol{C} \in \mathrm{CS}_{k}(\mathcal{G}(\mathcal{H}))} \frac{\mathrm{SW}\left(\boldsymbol{C}^{*}\right)}{\mathrm{SW}(\boldsymbol{C})}$, and again we adopt the convention that $\operatorname{CPoA}_{k}(\mathcal{G}(\mathcal{H})):=1$ if $\mathcal{G}(\mathcal{H})$ does not admit $k$-core stable coalition structures.

If $\mathrm{NPoA}_{k}(\mathcal{G}(\mathcal{H}))=\infty\left(\right.$ resp. $\left.\operatorname{CPoA}_{k}(\mathcal{G}(\mathcal{H}))=\infty\right)$, we say that the $k$-Nash (resp. $k$-core) price of anarchy of $\mathcal{G}(\mathcal{H})$ is unbounded. Since $\operatorname{NS}_{k}(\mathcal{G}(\mathcal{H})) \subseteq \operatorname{CS}_{k}(\mathcal{G}(\mathcal{H}))$, we have that $\operatorname{NPoA}_{k}(\mathcal{G}(\mathcal{H})) \leq \mathrm{CPoA}_{k}(\mathcal{G}(\overline{\mathcal{H}}))$. Furthermore, given two integers $h$ and $k$ with $1 \leq h \leq k$, since each $k$-Nash stable (resp. $k$-core stable) coalition structure is also $h$-Nash stable (resp. $h$-core stable), we have that $\operatorname{NPoA}_{k}(\mathcal{G}(\mathcal{H})) \leq$ $\operatorname{NPoA}_{h}(\mathcal{G}(\mathcal{H}))\left(\right.$ resp. $\left.\mathrm{CPoA}_{k}(\mathcal{G}(\mathcal{H})) \leq \operatorname{CPoA}_{h}(\mathcal{G}(\mathcal{H}))\right)$.

\section{The $k$-core price of anarchy}

In this section, we show upper and lower bounds on the $k$ core price of anarchy of any standard $r$-HHG with $k \geq r$, which are asymptotically tight in the variable $\rho:=\bar{n} / k$.

\footnotetext{
${ }^{1}$ We set the price of anarchy equal to 1 (that is the lowest possible value), in such a way that instances not admitting stable coalition structures are implicitly discarded when computing the price of anarchy of an arbitrary HHG.
} 
Furthermore, we show that, if either the considered $r$ hypergraph is not standard, or $k<r$, there exists an $r$-HHG with unbounded $k$-core price of anarchy.

Theorem 1 (Upper Bound). For any standard $r-H H G \mathcal{G}(\mathcal{H})$ with $n$ agents, and for any integer $k \geq r$, we have that

$$
\mathrm{CPoA}_{k}(\mathcal{G}(\mathcal{H})) \leq \frac{r(n-1)_{r-1}}{(k-1)_{r-1}}
$$

Proof. Let $\mathcal{G}(\mathcal{H})$ be a standard $r$-HHG, $\boldsymbol{C}=$ $\left\{C_{1}, C_{2}, \ldots, C_{h}\right\}$ be a $k$-core stable coalition structure of $\mathcal{G}(\mathcal{H})$ minimizing the social welfare, and $\boldsymbol{C}^{*}=\left\{C_{1}^{*}, C_{2}^{*}, \ldots, C_{h^{*}}^{*}\right\}$ be a social optimum of $\mathcal{G}(\mathcal{H})$. We assume w.l.o.g. that the considered $r$-hypergraph $\mathcal{H}$ is complete. Indeed, if it is not the case, it suffices to add dummy hyperedges with null weights to obtain an equivalent strategic game based on a complete hypergraph.

By definition of $k$-core stable coalition structure, given $Z \subseteq V$ with $|Z|=k$, there exists at least one agent $i_{1}^{Z} \in Z$ such that $u_{i_{1}^{Z}}(\boldsymbol{C}) \geq u_{i_{1}^{Z}}\left(\boldsymbol{C} \mid Z \cap C^{*}\left(i_{1}^{Z}\right)\right)$. This fact holds since, given a coalition $C_{l}^{*}$ of the social optimum such that $C_{l}^{*} \cap Z \neq \emptyset$, we have that $\left|C_{l}^{*} \cap Z\right| \leq k$, and thus, by definition of $k$-core stable coalition structure, there exists an agent $i_{1}^{Z} \in C_{l}^{*} \cap Z \subseteq Z$ such that $u_{i_{1}^{Z}}(\boldsymbol{C}) \geq$ $u_{i_{1}^{Z}}\left(\boldsymbol{C} \mid Z \cap C_{l}^{*}\right)=u_{i_{1}^{Z}}\left(\boldsymbol{C} \mid Z \cap C^{*}\left(i_{1}^{Z}\right)\right)$, that is, agent $i_{1}^{Z}$ does not improve her utility after forming a new coalition with all the agents of $Z \cap C_{l}^{*}$. We denote agent $i_{1}^{Z}$ as 1 -leader of $Z$. Let $Z_{2}:=Z_{1} \backslash\left\{i_{1}^{Z}\right\}$. Again by definition of $k$-core stable coalition structure, there exists at least one agent $i_{2}^{Z} \in Z_{2}$ such that $u_{i_{2}^{Z}}(\boldsymbol{C}) \geq u_{i_{2}^{Z}}\left(\boldsymbol{C} \mid Z_{2} \cap C^{*}\left(i_{2}^{Z}\right)\right)$, that is, agent $i_{2}^{Z}$ does not improve her utility after forming a new coalition with all the other agents of $Z_{2} \cap C^{*}\left(i_{2}^{Z}\right)$. We denote agent $i_{2}^{Z}$ as 2-leader of $Z$. By proceeding iteratively, we get that, for any $j \in[k]$, there exists an agent $i_{j}^{Z} \in Z_{j}$, called $j$-leader of $Z$, such that $u_{i_{j}^{Z}}(\boldsymbol{C}) \geq u_{i_{j}^{Z}}\left(\boldsymbol{C} \mid Z_{j} \cap C^{*}\left(i_{j}^{Z}\right)\right)$, where $Z_{1}:=Z$, and $Z_{j}:=Z_{j-1} \backslash\left\{i_{j-1}^{Z}\right\}=Z \backslash\left\{i_{1}^{Z}, i_{2}^{Z}, \ldots, i_{j-1}^{Z}\right\}$ if $j \geq 2$. Thus, by summing the previous inequalities over all $j \in[k]$ and $Z \subseteq V$ such that $|Z|=k$, we get

$\sum_{Z \subseteq V:|Z|=k} \sum_{j=1}^{k} u_{i_{j}^{Z}}(\boldsymbol{C}) \geq \sum_{Z \subseteq V:|Z|=k} \sum_{j=1}^{k} u_{i_{j}^{Z}}\left(\boldsymbol{C} \mid Z_{j} \cap C^{*}\left(i_{j}^{Z}\right)\right)$

We exploit the following two lemmas.

$\begin{array}{ll}\text { Lemma 1. } \sum_{Z \subseteq V:|Z|=k} \sum_{j=1}^{k} u_{i_{j}^{Z}}(\boldsymbol{C}) & = \\ \left(\begin{array}{c}n-1 \\ k-1\end{array}\right) \sum_{s=1}^{r}\left(s \sum_{l=1}^{h} \sum_{e \in E\left(C_{l}\right):|e|=s} w(e)\right) . & =\end{array}$

Proof of Lemma 1. We have that

$$
\begin{aligned}
\sum_{j=1}^{k} u_{i_{j}^{Z}}(\boldsymbol{C}) & =\sum_{j=1}^{k} \sum_{e \in E\left(C\left(i_{j}^{Z}\right)\right): i_{j}^{Z} \in e} w(e) \\
& =\sum_{l=1}^{h} \sum_{e \in E\left(C_{l}\right)}|e \cap Z| w(e)
\end{aligned}
$$

where (3) holds since, given a coalition $C_{l}$ and a hyperedge $e \subseteq C_{l}$, we have that each hyperedge $e$ appearing in (2) is counted as many times as the number of leaders contained in $e$, that is $|e \cap Z|$. Now, by summing (3) over all the possible subsets $Z \subseteq V$ of $k$ agents, we get

$$
\begin{aligned}
& \sum_{Z \subseteq V:|Z|=k} \sum_{j=1}^{k} u_{i_{j}^{Z}}(\boldsymbol{C})=\sum_{Z \subseteq V:|Z|=k} \sum_{l=1}^{h} \sum_{e \in E\left(C_{l}\right)}|e \cap Z| w(e) \\
& =\sum_{l=1}^{h} \sum_{e \in E\left(C_{l}\right)} w(e)\left(\sum_{Z \subseteq V:|Z|=k}|e \cap Z|\right) \\
& =\sum_{l=1}^{h} \sum_{e \in E\left(C_{l}\right)} w(e)\left(\sum_{Z \subseteq V:|Z|=k} \sum_{i \in e \cap Z} 1\right) \\
& =\sum_{l=1}^{h} \sum_{e \in E\left(C_{l}\right)} w(e)\left(\sum_{i \in e} \sum_{Z \subseteq V:|Z|=k, i \in Z} 1\right) \\
& =\sum_{l=1}^{h} \sum_{e \in E\left(C_{l}\right)} w(e)\left(\sum_{i \in e}\left|\left\{Z^{\prime} \subseteq V \backslash\{i\}:\left|Z^{\prime}\right|=k-1\right\}\right|\right) \\
& =\sum_{l=1}^{h} \sum_{e \in E\left(C_{l}\right)} w(e)\left(\sum_{i \in e}\left(\begin{array}{l}
n-1 \\
k-1
\end{array}\right)\right) \\
& =\sum_{s=1}^{r}\left(\sum_{l=1}^{h} \sum_{e \in E\left(C_{l}\right):|e|=s} w(e)\left(\sum_{i \in e}\left(\begin{array}{l}
n-1 \\
k-1
\end{array}\right)\right)\right) \\
& =\sum_{s=1}^{r}\left(\sum_{l=1}^{h} \sum_{e \in E\left(C_{l}\right):|e|=s} w(e)\left(\begin{array}{l}
n-1 \\
k-1
\end{array}\right) s\right),
\end{aligned}
$$

and this shows the claim.

Lemma 2. $\sum_{Z \subseteq V:|Z|=k} \sum_{j=1}^{k} u_{i_{j}^{Z}}\left(\boldsymbol{C} \mid Z_{j} \cap C^{*}\left(i_{j}^{Z}\right)\right)=$ $\sum_{s=1}^{r} \frac{1}{s}\left(\begin{array}{l}n-s \\ k-s\end{array}\right)\left(s \sum_{l=1}^{h^{*}} \sum_{e \in E\left(C_{l}^{*}\right):|e|=s} w(e)\right)$.

Proof of Lemma 2. We get

$$
\begin{aligned}
\sum_{j=1}^{k} u_{i_{j}^{Z}}\left(\boldsymbol{C} \mid Z_{j} \cap C^{*}\left(i_{j}^{Z}\right)\right) & =\sum_{j=1}^{k} \sum_{e \in E\left(Z_{j} \cap C^{*}\left(i_{j}^{Z}\right)\right): i_{j}^{Z} \in e} w(e) \\
& =\sum_{l=1}^{h^{*}} \sum_{e \in E\left(Z \cap C_{l}^{*}\right)} w(e),
\end{aligned}
$$

where (5) holds since, in (4), the unique hyperedges that are counted are those belonging to $E\left(Z \cap C_{l}^{*}\right)$ for some $l \in\left[h^{*}\right]$, and each of these hyperedges is counted exactly once. By summing (5) over all the subsets $Z \subseteq V$ of $k$ agents, we get

$$
\begin{aligned}
& \sum_{Z \subseteq V:|Z|=k} \sum_{j=1}^{k} u_{i_{j}^{Z}}\left(\boldsymbol{C} \mid Z_{j} \cap C^{*}\left(i_{j}^{Z}\right)\right) \\
= & \sum_{Z \subseteq V:|Z|=k} \sum_{l=1}^{h^{*}} \sum_{e \in E\left(Z \cap C_{l}^{*}\right)} w(e) \\
= & \sum_{l=1}^{h^{*}} \sum_{Z \subseteq V:|Z|=k} \sum_{e \in E\left(Z \cap C_{l}^{*}\right)} w(e) \\
= & \sum_{l=1}^{h^{*}} \sum_{e \in E\left(C_{l}^{*}\right)} w(e) \cdot|\{Z \subseteq V:|Z|=k, e \subseteq Z\}| .
\end{aligned}
$$


Given a coalition $C_{l}^{*}$ and a hyperedge $e \in E\left(C_{l}^{*}\right)$, we have that $|\{Z \subseteq V:|Z|=k, e \subseteq Z\}|$ is equal to the number of ways to select $k-|e|$ elements within set $V \backslash e$, that is $\left(\begin{array}{l}n-|e| \\ k-|e|\end{array}\right)$. Thus, by continuing from (7), we get

$$
\begin{aligned}
& \sum_{l=1}^{h^{*}} \sum_{e \in E\left(C_{l}^{*}\right)} w(e) \cdot|\{Z \subseteq V:|Z|=k, e \subseteq Z\}| \\
& =\sum_{l=1}^{h^{*}} \sum_{e \in E\left(C_{l}^{*}\right)} w(e)\left(\begin{array}{l}
n-|e| \\
k-|e|
\end{array}\right) \\
& =\sum_{s=1}^{r}\left(s \sum_{l=1}^{h^{*}} \sum_{\substack{e \in E\left(C_{l}^{*}\right): \\
|e|=s}} w(e)\right) \frac{1}{s}\left(\begin{array}{l}
n-s \\
k-s
\end{array}\right),
\end{aligned}
$$

and the claim follows.

By (1), Lemma 1, and Lemma 2, we get

$$
\begin{aligned}
& \sum_{s=1}^{r} \overbrace{\left(\begin{array}{l}
n-1 \\
k-1
\end{array}\right)}^{\lambda} \overbrace{\left(s \sum_{l=1}^{h} \sum_{e \in E\left(C_{l}\right):|e|=s} w(e)\right)}^{x_{s}} \\
& =\sum_{\substack{Z \subseteq V: \\
|Z|=k}} \sum_{j=1}^{k} u_{i_{j}^{Z}}(\boldsymbol{C}) \geq \sum_{\substack{Z \subseteq V: j \\
|Z|=k}} \sum_{j=1}^{k} u_{i_{j}^{Z}}\left(\boldsymbol{C} \mid Z_{j} \cap C^{*}\left(i_{j}^{Z}\right)\right)
\end{aligned}
$$

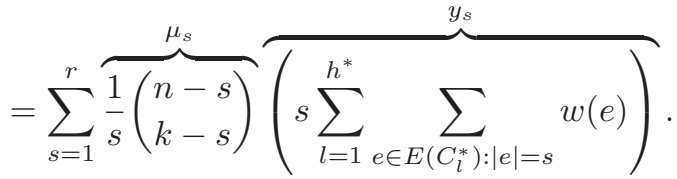

Now, we have the following lemmas. The proof of Lemma 3 uses the hypothesis that the considered game is standard.

Lemma 3. $y_{s} \geq 0$ for any $s \in[r]$.

Lemma 4. $\min _{s \in[r]} \mu_{s}=\mu_{r}$.

By (9), we get $\lambda \cdot \operatorname{SW}(\boldsymbol{C})=\lambda \sum_{s \in[r]} x_{s}=$ $\sum_{s \in[r]} \lambda x_{s} \geq \sum_{s \in[r]} \mu_{s} y_{s} \geq\left(\min _{s \in[r]} \mu_{s}\right) \sum_{s \in[r]} y_{s}=$ $\left(\min _{s \in[r]} \mu_{s}\right) \operatorname{SW}\left(C^{*}\right)$, where the second last inequality holds because of Lemma 3 and 4 . Thus, we get $\frac{\operatorname{sW}\left(C^{*}\right)}{\operatorname{SW}(\boldsymbol{C})}=$ $\frac{\sum_{s=1}^{r} y_{s}}{\sum_{s=1}^{r} x_{s}} \leq \frac{\lambda}{\min _{s \in[r]} \mu_{s}}=\frac{\lambda}{\mu_{r}}=\frac{\left(\begin{array}{c}n-1 \\ k-1\end{array}\right)}{\frac{1}{r}\left(\begin{array}{l}n-r \\ k-r\end{array}\right)}=\frac{r(n-1)_{r-1}}{(k-1)_{r-1}}$, and this concludes the proof.

The following corollary holds.

Corollary 1. The upper bound shown in Theorem 1 holds for any weakly uniform $\mathrm{r}-\mathrm{HHG}$ and for any $r-H H G$ with non-negative weights.

We now show an almost tight lower bound, holding even for non-negative weights.

Theorem 2. For any integers $r \geq 2, k \geq r$, and $n \geq k$, there exists an $r$-HHG $\mathcal{G}(\mathcal{H})$ with non-negative weights such that $\mathrm{CPoA}_{k}(\mathcal{G}(\mathcal{H})) \geq \frac{n(n-1)_{r-1}}{k\lfloor n / k\rfloor(k-1)_{r-1}+k^{\prime}\left(k^{\prime}-1\right)_{r-1}}$, where $k^{\prime}:=n-k\lfloor n / k\rfloor$, and $\left(k^{\prime}-1\right)_{r-1}:=0$ if $k^{\prime}<r$.
Proof sketch. Consider an $r$-HHG $\mathcal{G}(\mathcal{H})$, where $\mathcal{H}$ is a complete and uniform $r$-hypergraph with $n$ nodes, and each hyperedge has unitary weight. Let $\boldsymbol{C}$ be the coalition structure in which one coalition $C^{\prime}$ has $k^{\prime}=n-k\lfloor n / k\rfloor$ agents, and $\lfloor n / k\rfloor$ coalitions have each $k$ agents, and let $\boldsymbol{C}^{*}$ be the grand coalition structure. One can easily observe that $C$ is $k$-core stable. Finally, we show that $\mathrm{CPoA}_{k}(\mathcal{G}(\mathcal{H})) \geq \frac{\mathrm{SW}\left(\boldsymbol{C}^{*}\right)}{\mathrm{SW}(\boldsymbol{C})}=$ $\frac{n(n-1)_{r-1}}{k\lfloor n / k\rfloor(k-1)_{r-1}+k^{\prime}\left(k^{\prime}-1\right)_{r-1}}$.

The following theorem provides a better lower bound when $k$ is close to $n$, holding even for the $k$-Nash price of anarchy and if $k \geq r-1$.

Theorem 3. For any integers $r \geq 2, k \geq r-1$, and $n \geq$ $\max \{k, r\}$, there exists an $r-H H G \mathcal{G}(\mathcal{H})$ with non-negative weights such that $\mathrm{CPoA}_{k}(\mathcal{G}(\mathcal{H})) \geq \operatorname{NPoA}_{k}(\mathcal{G}(\mathcal{H})) \geq 1+$ $\frac{(\lfloor n / 2\rfloor-1)_{r-1}}{2(\min \{k,\lfloor n / 2\rfloor-1\})_{r-1}}$.

Remark 3. If $r$ is constant w.r.t. the input instance, the upper bound of Theorem 1 and the lower bounds of Theorem 2 and 3 are asymptotically tight in variable $\rho:=n / k$, and their value increase as $\Theta\left(\rho^{r-1}\right)$.

In the following theorems we show that, without the assumptions done in the Theorem 1, there always exists a HHG with unbounded $k$-core price of anarchy.

Theorem 4. For any integers $r \geq 2, k<r$, and $n \geq r$, there exists an $r-H H G \mathcal{G}(\mathcal{H})$ with non-negative weights and $n$ agents such that $\mathrm{CPoA}_{k}(\mathcal{G}(\mathcal{H}))=\infty$.

Proof sketch. We consider an $r$-HHG $\mathcal{G}(\mathcal{H})$ made of a unique hyperedge of weight 1 and arity $r$. We show that the singleton coalition structure $C$ is $k$-core stable and $\operatorname{SW}\left(C^{*}\right)=0$, and the grand coalition structure $\boldsymbol{C}^{*}$ verifies $\operatorname{SW}\left(\boldsymbol{C}^{*}\right)=n$, thus $\mathrm{CPoA}_{k}(\mathcal{G}(\mathcal{H})) \geq \frac{\operatorname{sW}\left(\boldsymbol{C}^{*}\right)}{\operatorname{SW}(\boldsymbol{C})}=\infty$.

Theorem 5. For any integers $r \geq 3, k \geq r$, and $n \geq k$, there exists a (non-standard) $r-\bar{H} H G \mathcal{G}(\overline{\mathcal{H}})$ with $n$ agents such that $\mathrm{NPoA}_{k}(\mathcal{G}(\mathcal{H}))=\mathrm{CPoA}_{k}(\mathcal{G}(\mathcal{H}))=\infty .^{2}$

Proof sketch. Let $\mathcal{H}$ be a weighted hypergraph with $k$ nodes, containing only all the hyperedges of arity $r$ and 2 that contain both nodes 1 and 2. Each hyperedge with arity $r$ has weight $w_{r}=1$, and the unique hyperedge with arity 2 has weight $w_{2}=-\frac{k-2}{r-2}$. We show that the singleton coalition structure $\boldsymbol{C}$ is $k$-Nash stable and $\operatorname{SW}(\boldsymbol{C})=0$, and that the grand coalition structure $C^{*}$ verifies $\operatorname{SW}\left(C^{*}\right)>0$. Thus, $\mathrm{CPoA}_{k}(\mathcal{G}(\mathcal{H})) \geq \operatorname{NPoA}_{k}(\mathcal{G}(\mathcal{H})) \geq \frac{\operatorname{SW}\left(\boldsymbol{C}^{*}\right)}{\operatorname{SW}(\boldsymbol{C})}=\infty$.

\section{The $k$-Nash price of anarchy}

We now show upper and lower bounds on the $k$-Nash price of anarchy. As $\operatorname{NPoA}_{k}(\mathcal{G}(\mathcal{H})) \leq \mathrm{CPoA}_{k}(\mathcal{G}(\mathcal{H}))$ for any game $\mathcal{G}(\mathcal{H})$, we get the following corollary of Theorem 1 .

Corollary 2. The upper bound shown in Theorem 1 holds also for the $k$-Nash price of anarchy.

\footnotetext{
${ }^{2}$ We do not consider the case $r=2$, since all graph hedonic games are standard.
} 
If weights are non-negative, we have the following slightly better upper bound, holding also for $k=r-1$.

Theorem 6. For any $r-H H G \mathcal{G}(\mathcal{H})$ with non-negative weights and $n$ agents, and for any integer $k$ such that $n-1 \geq$ $k \geq r-1$, we have that ${ }^{3}$

$$
\mathrm{NPoA}_{k}(\mathcal{G}(\mathcal{H})) \leq \frac{\frac{(n-1) !}{(k-1) !}}{\frac{\chi_{[k]}(r) n(n-r-1) !}{r(k-r) !}+\frac{(n-r) !}{(k+1-r) !}} .
$$

Proof sketch. Let $\mathcal{G}(\mathcal{H})$ be a standard $r-\mathrm{HHG}$, and let $\boldsymbol{C}=$ $\left\{C_{1}, C_{2}, \ldots, C_{h}\right\}$ be a $k$-Nash stable coalition structure of $\mathcal{G}(\mathcal{H})$ having minimum social welfare. Observe that, as the weights are non-negative, the grand coalition $C^{*}$ is a social optimum. We assume w.l.o.g. that the considered $r$ hypergraph $\mathcal{H}$ is complete. Furthermore, we assume w.l.o.g. that all the hyperedges of arity 1 have null-weights (indeed, by removing them, the set of stable outcomes is not affected and the $k$-Nash price of anarchy does not decrease). By definition of $k$-Nash stable coalition structure, we have that, given $Z \subseteq V$ with $|Z|=k+1$ and $v \in Z$, there exists at least one agent $i_{1}^{Z, v} \in Z \backslash\{v\}$ such that $u_{i_{1}^{Z, v}}(\boldsymbol{C}) \geq u_{i_{1}^{Z}}\left(\boldsymbol{C} \mid \varphi^{Z, v}\right)$, where $\varphi^{Z, v}$ is the deviation function such that all the agents of $Z \backslash\{v\}$ move to coalition $C(v)$. As all the weights are non-negative, we get $u_{i_{1}^{Z, v}}(\boldsymbol{C}) \geq u_{i_{1}^{Z}}\left(\boldsymbol{C} \mid \varphi^{Z, v}\right) \geq u_{i_{1}^{Z}}(\boldsymbol{C} \mid Z)$. As in the proof of Theorem 1 , one can show that, for any $j \in[k]$, there exists an agent $i_{j}^{Z, v} \in Z_{j}^{v} \backslash\{v\}$, called $j$-leader of $(Z, v)$, such that $u_{i_{j}^{Z}}(\boldsymbol{C}) \geq u_{i_{j}^{Z}}\left(\boldsymbol{C} \mid Z_{j}^{v}\right)$, where $Z_{1}^{v}:=Z$, and $Z_{j}^{v}:=Z_{j-1}^{v} \backslash\left\{i_{j-1}^{Z, v}\right\}=Z \backslash\left\{i_{1}^{Z, v}, i_{2}^{Z, v}, \ldots, i_{j-1}^{Z, v}\right\}$ if $j \geq 2$. By considering a slightly more complicated proof than that of Theorem 1, we show that

$$
\begin{aligned}
& \sum_{s=2}^{r} \overbrace{\left(\begin{array}{l}
n-2 \\
k-1
\end{array}\right)(n-1)}^{\lambda} \overbrace{s \sum_{l=1}^{h} \sum_{e \in E\left(C_{l}\right):|e|=s} w(e)}^{x_{s}} \\
& =\sum_{\substack{(Z, v): Z \subseteq V, j \\
|Z|=k+1, v \in Z}} \sum_{j=1}^{k} u_{i_{j}^{Z, v}}(\boldsymbol{C}) \geq \sum_{\substack{(Z, v): Z \subseteq V, j=1 \\
|Z|=k+1, v \in Z}} \sum_{j}^{k} u_{i_{j}^{Z, v}}\left(\boldsymbol{C} \mid Z_{j}^{v}\right) \\
& =\sum_{s=2}^{r} \overbrace{\left(\frac{\chi_{[k]}(s) n\left(\begin{array}{c}
n-s-1 \\
k-s
\end{array}\right)}{s}+\left(\begin{array}{c}
n-s \\
k+1-s
\end{array}\right)\right)}^{\mu_{s}} \overbrace{s \sum_{\substack{e \in E: \\
|e|=s}} w(e)}^{y_{s}},
\end{aligned}
$$

implying that $\frac{\operatorname{SW}\left(\boldsymbol{C}^{*}\right)}{\mathrm{SW}(\boldsymbol{C})}=\frac{\sum_{s=2}^{r} y_{s}}{\sum_{s=2}^{r} x_{s}} \leq \frac{\lambda}{\min _{s \in[r] \backslash\{1\}} \mu_{s}}=\frac{\lambda}{\mu_{r}}$, and then showing the claim.

Remark 4. If we consider $r$ as a constant, the upper bounds shown in Corollary 2, Theorem 3, and the lower bound shown in Theorem 3 , are asymptotically tight in $\rho:=n / k$, and their value increase as $\Theta\left(\rho^{r-1}\right)$.

\footnotetext{
${ }^{3}$ We consider the case $k \leq n-1$ only, since for $k=n$ we have that $\operatorname{NPoA}_{n-1}(\mathcal{G}(\mathcal{H}))$ is an upper bound for $\operatorname{NPoA}_{k}(\mathcal{G}(\mathcal{H}))$.
}

Because of Theorem 5 and the following results, we have that, without the assumptions done in Corollary 2 and Theorem 6 , in most of the cases there exists a HHG with $\mathrm{NPoA}_{k}=\infty$. We do not consider the case of standard $r$ HHG with $k=r-1$ only, that is left as open problem.

Theorem 7. For any integers $r \geq 3, k<r-1$, and $n \geq r$, there exists an $r-H H G \mathcal{G}(\mathcal{H})$ with non-negative weights and $n$ agents such that $\operatorname{NPoA}_{k}(\mathcal{G}(\mathcal{H}))=\infty$.

Theorem 8. For any integers $r \geq 3$ and $n \geq r$, if $k=r-1$, there exists a non-standard $r-H H G \mathcal{G}(\mathcal{H})$ with $n$ agents such that $\mathrm{NPoA}_{k}(\mathcal{G}(\mathcal{H}))=\infty$.

\section{Computational issues}

In this section, we consider the computational complexity of evaluating the inefficiency of a given $r$-HHG. In particular, we consider the problems $r$-Core-PoA and r-Nash-PoA, defined as follows: given an integer $k \geq 2$, an $r$-HHG $\mathcal{G}(\mathcal{H})$, and a rational number $M \geq 1$, r-Core-PoA (resp. r-NashPoA) asks to check whether the $k$-core (resp. $k$-Nash) price of anarchy of $\mathcal{G}(\mathcal{H})$ is lower than $M$. We show that such problems are computationally hard.

Theorem 9. r-Core-PoA is NP-hard, even when considering instances with non-negative weights.

Proof sketch. Polynomial-time reduction to the minimum maximal matching problem (MMS) (Yannakakis and Gavril 1980). We transform the input unweighed graph $\mathcal{H}$ of MMS into the corresponding graph hedonic game $\mathcal{G}(\mathcal{H})$, and we show that there always exists a worst-case 2-core stable outcome of $\mathcal{G}(\mathcal{H})$ having the structure of a maximal matching with minimum cardinality.

Theorem 10. r-Nash-PoA is NP-hard, even when considering instances with non-negative weights.

Proof sketch. Polynomial-time reduction to the max clique problem (Karp 1972).

\section{Future works}

Our work leaves several research directions. First of all, despite the fact that our lower and upper bounds are asymptotically tight, it would be nice to reduce their constant multiplicative gap. Furthermore, it remains to analyse the $k$-Nash price of anarchy of standard $r$-HHGs with $k=r-1$. It would be interesting to study hypergraph variants of other coalition formation games (such as fractional hedonic games and max-cut games), and the inefficiency under different stability criteria (e.g., contractual Nash stability, Pareto optimality, etc.). It would be also nice to investigate how much some restrictions on the hypergraph topology affect the performance. Finally, we believe that the computational problems studied in this paper are $\Sigma_{2}^{p}$-complete, and we leave this conjecture as another worth investigating open question.

\section{Acknowledgments}

This work was partially supported by the Italian MIUR PRIN 2017 Project ALGADIMAR "Algorithms, Games, and Digital Markets". We would like to thank the anonymous reviewers for their suggestions and comments. 


\section{References}

Apt, K. R.; de Keijzer, B.; Rahn, M.; Schäfer, G.; and Simon, S. 2017. Coordination games on graphs. Int. J. Game Theory 46(3):851-877.

Aziz, H.; Brandl, F.; Brandt, F.; Harrenstein, P.; Olsen, M.; and Peters, D. 2019. Fractional hedonic games. ACM Trans. Econ. Comput. 7(2):6:1-6:29.

Aziz, H.; Brandt, F.; and Seedig, H. G. 2011. Optimal partitions in additively separable hedonic games. In IJCAI'11, 43-48.

Aziz, H.; Brandt, F.; and Seedig, H. G. 2013. Computing desirable partitions in additively separable hedonic games. Artificial Intelligence 195:316 - 334.

Balliu, A.; Flammini, M.; Melideo, G.; and Olivetti, D. 2019. On non-cooperativeness in social distance games. J. Artif. Intell. Res. 66:625-653.

Balliu, A.; Flammini, M.; and Olivetti, D. 2017. On pareto optimality in social distance games. In AAAI'17, 349-355.

Banerjee, S.; Konishi, H.; and Sönmez, T. 2001. Core in a simple coalition formation game. Social Choice and Welfare 18(1):135153.

Bilò, V.; Fanelli, A.; Flammini, M.; Monaco, G.; and Moscardelli, L. 2019. Optimality and nash stability in additive separable generalized group activity selection problems. In IJCAI'19, 102-108.

Bilò, V.; Fanelli, A.; Flammini, M.; Monaco, G.; and Moscardelli, L. 2018. Nash stable outcomes in fractional hedonic games: Existence, efficiency and computation. J. Artif. Int. Res. 62(1):315-371.

Bogomolnaia, A., and Jackson, M. O. 2002. The stability of hedonic coalition structures. Games and Economic Behavior 38(2):201 -230 .

Cai, Y., and Daskalakis, C. 2011. On minmax theorems for multiplayer games. In SODA'11, 217-234.

Carosi, R.; Fioravanti, S.; Gualà, L.; and Monaco, G. 2019. Coalition resilient outcomes in max k-cut games. In SOFSEM'19, 94107.

Carosi, R.; Monaco, G.; and Moscardelli, L. 2019. Local core stability in simple symmetric fractional hedonic games. In $A A$ MAS'19, 574-582.

Chalkiadakis, G.; Elkind, E.; and Wooldridge, M. J. 2011. Computational Aspects of Cooperative Game Theory. Synthesis Lectures on Artificial Intelligence and Machine Learning. Morgan \& Claypool Publishers.

Darmann, A.; Elkind, E.; Kurz, S.; Lang, J.; Schauer, J.; and Woeginger, G. 2018. Group activity selection problem with approval preferences. Int. J. Game Theory 47(3):767-796.

Deng, X., and Papadimitriou, C. H. 1994. On the complexity of cooperative solution concepts. Mathematics of Operations Research 19(2):257-266.

Drèze, J. H., and Greenberg, J. 1980. Hedonic coalitions: Optimality and stability. Econometrica 48(4):987-1003.

Elkind, E.; Fanelli, A.; and Flammini, M. 2016. Price of pareto optimality in hedonic games. In AAAI'16, 475-481.

Feldman, M., and Friedler, O. 2015. A unified framework for strong price of anarchy in clustering games. In ICALP'15, 601613.

Flammini, M.; Monaco, G.; Moscardelli, L.; Shalom, M.; and Zaks, S. 2018. Online coalition structure generation in graph games. In AAMAS'18, 1353-1361.

Flammini, M.; Monaco, G.; and Zhang, Q. 2017. Strategyproof mechanisms for additively separable hedonic games and fractional hedonic games. In WAOA'17, 301-316.
Gairing, M., and Savani, R. 2010. Computing stable outcomes in hedonic games. In SAGT'10, 174-185.

Gourvès, L., and Monnot, J. 2009. On strong equilibria in the max cut game. In WINE'09, 608-615.

Gourvès, L., and Monnot, J. 2010. The max $k$-cut game and its strong equilibria. In TAMC'10, 234-246.

Igarashi, A.; Bredereck, R.; and Elkind, E. 2017. On parameterized complexity of group activity selection problems on social networks. In AAMAS'17, 1575-1577.

Igarashi, A.; Peters, D.; and Elkind, E. 2017. Group activity selection on social networks. In AAAI'17, 565-571.

Kaklamanis, C.; Kanellopoulos, P.; and Papaioannou, K. 2016. The price of stability of simple symmetric fractional hedonic games. In SAGT'16, 220-232.

Karp, R. M. 1972. Reducibility among combinatorial problems. In Complexity of Computer Computations.

Koutsoupias, E., and Papadimitriou, C. 1999. Worst-case equilibria. In STACS'99, 404-413.

Monaco, G.; Moscardelli, L.; and Velaj, Y. 2018. Stable outcomes in modified fractional hedonic games. In AAMAS'18, 937-945.

Monaco, G.; Moscardelli, L.; and Velaj, Y. 2019. On the performance of stable outcomes in modified fractional hedonic games with egalitarian social welfare. In AAMAS'19, 873-881.

Olsen, M. 2009. Nash stability in additively separable hedonic games and community structures. Theory Comput. Syst. 45(4):917-925.

Olsen, M. 2012. On defining and computing communities. In CATS'12, 97-102.

Osborne, M. 2004. An introduction to game theory. Oxford Univ. Press.

Peters, D., and Elkind, E. 2015. Simple causes of complexity in hedonic games. In IJCAI'15, 617-623.

Rahn, M., and Schäfer, G. 2015. Efficient equilibria in polymatrix coordination games. In MFCS'15, 529-541.

Simon, S., and Wojtczak, D. 2017. Synchronisation games on hypergraphs. In IJCAI'17, 402-408.

Sung, S.-C., and Dimitrov, D. 2010. Computational complexity in additive hedonic games. European Journal of Operational Research 203(3):635 - 639 .

Yannakakis, M., and Gavril, F. 1980. Edge dominating sets in graphs. SIAM J. Appl. Math. 38:364-372.

Yanovskaya, E. B. 1968. Equilibrium points in polymatrix games. Latvian Mathematical Collection 8(3):381-384. 